\title{
Transient Voltage Stability Probabilistic Assessment Based on Second-order Approximation of Stability Boundary
}

\author{
Xiaoying Zhang ${ }^{1, a}$, Bin $\mathrm{Fu}^{1, \mathrm{~b}}$ and Kun Wang ${ }^{1, \mathrm{c}}$ \\ ${ }^{1}$ College of Electrical and Information Engineering, Lanzhou University of Technology, Lanzhou \\ a245659219@qq.com, bfbin001@126.com, c471943808@qq.com
}

\begin{abstract}
Keywords: transient voltage stability; dynamic security region; probabilistic assessment
Abstract: Probabilistic assessment of power system transient voltage stability is a useful complement to determinate evaluation. The linear boundary of dynamic security region of the controlling unstable equilibrium point was determined on the basis of dynamical system theory. Consequently, power system transient voltage stability probabilistic model was established. The model can effectively consider many uncertain factors, such as generator output and internal potential, load active power and reactive power, fault location, fault type, fault-clearing time, etal. The simulation of New England 39 bus test system shows that the proposed assessment strategy is effective and accurate.
\end{abstract}

\section{Introduction}

Deterministic transient voltage stability assessment results are often too conservative, which can not fully consider the various uncertainties, and may ignore the individual extreme cases[1-2]. Especially in the competitive market conditions, the power sector needs to know the risk level of the system running, so as to provide the corresponding services according to user's requirements. Therefore, the deterministic analysis can not meet the needs of electric power enterprises. Thus, the power system transient voltage stability probability assessment is a useful supplement to the deterministic assessment.

In addition, the power system transient voltage stability analysis method is in the system of component parameters, operating conditions and disturbances are given, which belongs to the category of deterministic analysis. But there are many uncertainties in the practical power system operation, such as load fluctuation, fault type, fault location and fault clearing time[3-6]. Because of the uncertainty of the system parameters, it is difficult to give a conclusion that whether the system is stable or not, and the probability of the system is more practical. Therefore, from the point of view of probability, the impact of the uncertainty on the transient voltage stability of power system is properly evaluated. It is helpful to find out some serious operating conditions due to the randomness of the system, which is very likely to be neglected in the deterministic calculation. This is very important for improving the stability of power system. Therefore, this paper proposes a new method for transient voltage stability probability based on the two approaches of the stability region boundary.

\section{Transient voltage stability probability model}

The dynamic security region of the power system is a simple super polyhedron in $\mathrm{R}$ space, which consists of the planar and 1 or a few descriptions of the critical point of transient stability. The hyper plane can be expressed as:

$$
a^{T} u=\sum_{k=1}^{n} a_{k} u_{k}=c
$$


where, $u=\left[u_{1}, u_{2} \ldots, u_{n}\right]^{T}$ is a controllable variable for the transient stability of the system; $a=\left[a_{1}, a_{2} \ldots, a_{n}\right]^{T}$ is the coefficient of the super plane equation; is a constant that changes the state of the system.

Taking into account the controllable variables of generator and load nodes, the controllable variable is composed of mechanical power $P_{m i}$ and the internal electric potential $E_{i}$ of load node $i$, the active power $P_{L j}$ and reactive power $Q_{L j}$ of load node $j$. Therefore, Eq.1 can be rewritten as:

$$
\sum_{i \in G}\left(\alpha_{i} P_{m i}+\beta_{i} E_{i}\right)+\sum_{j \in L}\left(\eta_{j} P_{L j}+\lambda_{j} Q_{L j}\right)=c
$$

If the controllable variable satisfies the $\sum_{k=1}^{n} a_{k} u_{k} \leq c$, the controllable variable is located in the dynamic security region.It is considered that the system is transient stability when the accident E occurs.

$$
p(I \mid E)=p\left(u \notin \Omega_{d}\right)=p\left(\sum_{k=1}^{n} a_{k} u_{k}>c\right)
$$

Where, $I$ is the event that causes the system transient instability; $\Omega_{d}$ is a dynamic security region.

Edgeworth series expansion can effectively calculate the joint probability distribution of the random variables, which has been successfully applied to many problems. In order to Eq.3,the probability distribution function of joint random variable can be obtained by using the relationship between the semi invariants and the Edgeworth series coefficients:

$$
F(x)=\int_{x}^{+\infty} \varphi(x) d x+\varphi(x) \sum_{k=3}^{+\infty} C_{k} H_{k-1}(x)
$$

Where, $\varphi(x)$ is the probability density function of the normal distribution; $C$ is coefficients for Edgeworth series; $H_{k}(x)$ is k oder Hermite polynomial.

According to the Eq.3,the system transient instability probability can be obtained:

$$
p(I \mid E)=1-F(\bar{c})
$$

Where, Normalized random variable $\bar{c}=(c-m) / \sigma ; m$ and $\sigma$ are expected value and standard deviation of random variable.

The accident $\mathrm{E}$ contains fault line $\mathrm{W}$, fault location $\mathrm{X}$, fault type $\mathrm{Y}$ and fault clearing time $\mathrm{Z}$.Assume that $\mathrm{W}, \mathrm{X}, \mathrm{Y}$ and $\mathrm{Z}$ are independent of each other.

$$
p(E)=p(W X Y Z)=p(W) p(X) p(Y) p(Z)
$$

Generally speaking, the fault type can be divided into four categories (single phase to ground fault, two-phase to ground fault, interphase short circuit and three-phase short circuit), which is subject to the discrete probability distribution; The fault location of the line is generally not subject to uniform distribution, but the discrete probability distribution; the is subject to the normal distribution, and the parameters of these distributions can be determined by statistical analysis of historical data.

Therefore, the system transient instability probability is:

$$
p(I)=\sum_{i} \sum_{j} \sum_{k} \sum_{l}\left[p(I \mid E) p\left(W_{i} X_{j} Y_{k} Z_{l}\right)\right]
$$

Where, $W_{i}, X_{j}, Y_{k}, Z_{l}$ are the fault line, fault location, type of fault and fault clearing time. 


\section{Evaluation strategy}

In summary, the system transient stability probability assessment method can be summarized as the following four steps:

1. Seeking the controlling unstable equilibrium point;

2. Linear boundary for dynamic security region;

3. The transient instability probability $p(I \mid E)$ for a certain accident is calculated according to the Eq.5;

4. System transient instability probability $p(I)$ is calculated System transient instability probability $p(I)$.

\section{Numerical Example}

This paper uses New England 10-generator 39-bus test system showed in Fig.1

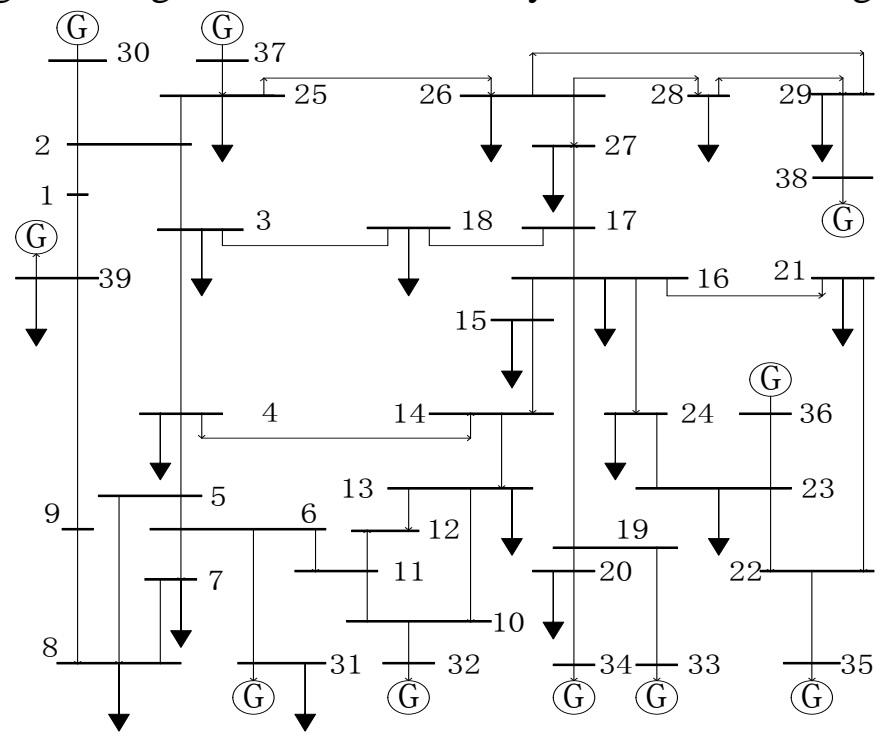

Fig.1 New England 10-generator 39-bus test system

A total of 12 lines are considered in the calculation. It is assumed that:

1. There is only one running state of the system.

2. The system is having three-phase short-circuit fault.

3. The probability of occurrence of each line is equal.

4. Consider that the line is divided into three parts, each part is replaced by a typical point $\left(Y_{1}, Y_{2}, \cdots \cdots\right)$, and the probability of each point of the line is proportional to the length of the line. Discrete probability density of the line fault location is shown in Fig.2.

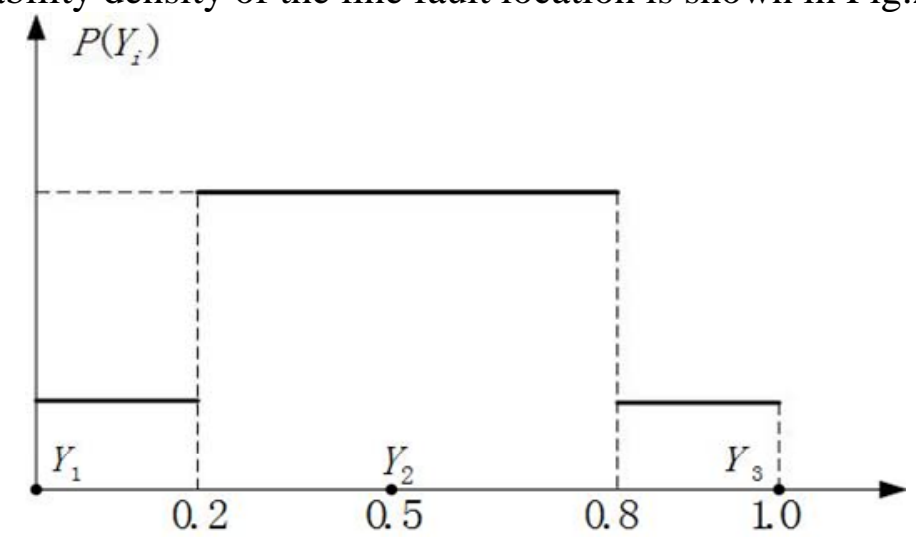

Fig.2 Discrete probabilistic distribution of fault location on the line

Table 1 shows the results of the transient voltage stability probability assessment. 
Tab.1 Probabilistic assessment results of transient voltage stability

\begin{tabular}{ccccc}
\hline $\begin{array}{c}\text { Fault } \\
\text { locatio } \\
n\end{array}$ & $\begin{array}{c}\text { Cut } \\
\text { line }\end{array}$ & \multicolumn{2}{c}{$\begin{array}{c}\text { Transient voltage } \\
\text { stability } \\
\text { probability }\end{array}$} & $\begin{array}{c}\text { Probabilit } \\
\text { y } \\
\text { calculation } \\
\text { error (\%) }\end{array}$ \\
\cline { 3 - 4 } & & $\begin{array}{c}\text { Time } \\
\text { domain } \\
\text { method }\end{array}$ & $\begin{array}{c}\text { Dynamic } \\
\text { security } \\
\text { region }\end{array}$ & \\
\hline 3 & $3-4$ & 0.6194 & 0.6225 & 0.500 \\
3 & $3-18$ & 0.6087 & 0.6164 & 1.265 \\
5 & $5-6$ & 0.5972 & 0.5987 & 0.258 \\
10 & $10-11$ & 0.5987 & 0.6072 & 1.419 \\
10 & $10-13$ & 0.5964 & 0.6018 & 0.905 \\
18 & $18-3$ & 0.6003 & 0.6110 & 1.783 \\
22 & $22-21$ & 0.5223 & 0.5152 & -1.359 \\
23 & $23-22$ & 0.5816 & 0.5620 & -3.370 \\
25 & $25-2$ & 0.5120 & 0.4960 & -3.125 \\
25 & $25-26$ & 0.5667 & 0.5557 & -1.941 \\
26 & $26-29$ & 0.4944 & 0.4888 & -1.132 \\
28 & $28-29$ & 0.4641 & 0.4602 & -0.084 \\
\hline
\end{tabular}

From Tab.1,when the system is in the generator outlet high voltage side of the three-phase short-circuit fault (line 25-2, line 25-26, line 23-22), the use of dynamic security region to obtain the transient voltage stability and time domain simulation method, the error of the $7 \%$ can meet the engineering requirements, other lines from the dynamic security region based on the transient voltage stability probability and time domain method are relatively small, can accurately estimate the transient voltage stability, indicating that this assessment method has a certain validity.

\section{Conclusion}

In this paper, a new method of transient voltage stability analysis is proposed, which is based on the analysis of transient voltage stability index and traditional probabilistic analysis method, which can be used to improve the efficiency of transient voltage stability analysis, and it is easy to be realized by computer. The effectiveness and accuracy of the proposed method are verified by the simulation of New England 10-generator 39-bus test system.

\section{References}

[1] Baosheng Tang: Power system Technology,vol.11(2003),p.1-6.In Chinese.

[2] Hongxi Zhang: Jiangsu Electrical Engineering,vol.2(2012),p.59-62. In Chinese.

[3] Taishan Xu,Yusheng XueandZhenxiang Han:Automation of Electric Power Systems,vol.20(1996),p.12-15.In Chinese.

[4] Yihong Wang,Shengwei Mei:Advanced Technology of Electrical Engineering and Energy, Vol.26(2006),p.39-44.In Chinese.

[5] QilongLiao,Yan Wei and Liu Huan:Automation of Electric Power Systems, Vol.3(2010),p.28-43. In Chinese.

[6] Changwang Yan,Xiaodong Zhang and Shuguang Liu:China Concrete And Cement Products, Vol.1(2014),p.49-52. In Chinese. 